\title{
Shoulder impairment and pain of individuals with newly acquired spinal cord injury compared to uninjured peers
}

\author{
Margaret Finley $^{1} \cdot$ Elizabeth Euiler $^{1} \cdot$ Thomas Trojian $^{2} \cdot$ Edward Gracely $^{3} \cdot$ Mary Schmidt-Read $^{4} \cdot$ Sara Kate Frye $^{5}$. \\ Marni Kallins ${ }^{5} \cdot$ Amanda Summers $^{5} \cdot$ Henry York $^{5,6} \cdot$ Paula Richley Geigle $^{7}$
}

Received: 18 June 2020 / Revised: 22 July 2020 / Accepted: 22 July 2020

(c) The Author(s), under exclusive licence to International Spinal Cord Society 2020

\begin{abstract}
Study design Cohort study.

Objectives Shoulder pain prevalence is high in those with spinal cord injury (SCI) and is associated with decreased function, participation restrictions and decreased quality of life. Limited evidence exists regarding physical impairments of newly acquired SCI. The current study compared musculoskeletal factors at rehabilitation initiation in individuals with newly acquired SCI to uninjured individuals. We hypothesized no impairment differences of shoulder pain, strength, mobility, muscle extensibility, or rotator cuff integrity would exist between groups.
\end{abstract}

Setting Multi-site laboratory setting.

Methods Thirty-five individuals with newly acquired SCI and age and gender-matched controls without SCI $(n=34)$ participated. Musculoskeletal Pain Survey, shoulder range of motion (ROM), strength, pectoralis minor muscle extensibility (PM) and tissue integrity [Ultrasound Pathology Rating Scale (USPRS)] were obtained.

Results Higher pain was reported by individuals experiencing new SCI along with lower strength across all bilateral measures, reduced elevation, external rotation, and horizontal adduction ROM, with large effect sizes. PM bilateral extensibility was reduced compared to controls, with moderate between group effect size; however, no USPRS score difference existed.

Conclusions This study provided the first comprehensive clinical description for individuals with newly acquired SCI. In comparison to matched uninjured controls, participants with new SCI reported greater shoulder pain with impairments in mobility, strength, and extensibility. The identified early clinical impairments aligned with progressive impairment including further pain development and persistence. Awareness and modification of these early clinical impairments may lead to improved long-term outcomes, improving the overall health and well-being of individuals with newly acquired SCI.

Sponsorship Spinal Cord Injury Research Program Investigator-Initiated Research Award under Award No. W81XWH-171-0476.

\section{Introduction}

In the United States over 273,00 people currently live with a spinal cord injury (SCI), with more than 12,500 newly

Margaret Finley

maf378@drexel.edu

1 Department of Physical Therapy and Rehabilitation Science, Drexel University, Philadelphia, PA, USA

2 Drexel University College of Medicine, Philadelphia, PA, USA

3 School of Public Health, Drexel University, Philadelphia, PA, USA acquired injuries annually [1]. Over $40 \%$ of individuals with SCI use a manual wheelchair for mobility [1]. Although manual wheelchair (MWC) use does promote and maintain physical fitness and greater independence, a significantly

4 Magee Rehabilitation Hospital/Jefferson Health, Philadelphia, PA, USA

5 University of Maryland Rehabilitation and Orthopaedic Institute, Baltimore, MD, USA

6 Department of Neurology, University Maryland School of Medicine, Baltimore, MD, USA

7 Department Physical Therapy, South College, Knoxville, TN, USA 
increased upper extremity demand may occur [2]. In addition to mobility, individuals with SCI use their upper extremities for transfers, pressure relief, and activities of daily living. Dependence on the upper extremities predisposes these individuals to debilitating upper limb impairments. Individuals with SCI who develop shoulder pain are more likely to experience a devastating loss of independence and quality of life (QoL), including restrictions in self-care, work, and leisure activities [3-5].

Prevalence of shoulder pain following SCI demonstrates a bimodal distribution: highest in the initial year and 15 or greater years post injury $[6,7]$. Research has investigated common clinical and biomechanical mechanisms of shoulder pain in those with chronic SCI [8-10]. However, evidence at the onset of SCI is limited to passive range of motion and gross muscle strength [11-13]. SCI is a sudden, unexpected event with average initial injury occurring at 43 years of age [1]. For individuals without SCI the incidence of asymptomatic shoulder tissue integrity change increases progressively with age [14]. Rotator cuff tendinopathy and fibrosis are seen in individuals 25-40 years of age with partial and full tears most common over 40 years of age [15]. Given SCI's acute onset, it is reasonable to assume pre-existing shoulder impairment exists, just as in the population without SCI.

Following SCI, individuals must increase strength for effective upper limb use during daily activities, transfers, and wheelchair propulsion. Increased upper extremity use following SCI places demand on an untrained musculoskeletal system or preexisting injured tissue, leading to acute injury differing from long-term overuse conditions common for people with chronic SCI. Shoulder pain within the 1st year of injury predicts chronic shoulder pain over an individual's lifetime [13].

To understand a biopsychosocial pain model, first we must understand the presenting clinical factors at injury onset. Therefore, our current purpose was to compare musculoskeletal factors in individuals with newly acquired $\mathrm{SCI}$ at the initiation of rehabilitation to an age and gendermatched group of uninjured individuals. We hypothesized no musculoskeletal impairment differences of shoulder pain, muscle strength, mobility, muscle extensibility, or rotator cuff integrity existed in a group of individuals with newly acquired SCI compared to a gender and age matched control group.

\section{Methods}

To support the aims of a larger longitudinal study, a crosssectional, multisite, repeated-measures design was employed with Institutional Review Board approval acquired from all sites. A-priori sample size analysis indicated to detect a meaningful difference $\left(\eta^{2}=0.3\right)$ in musculoskeletal factors across repeated measurements, 27 participants $(\alpha=0.05$, power $=80 \%$, Cohen's $d=0.70$ ) were needed. Considering $20 \%$ attrition, 35 individuals with newly acquired SCI were recruited and enrolled from inpatient rehabilitation programs at three rehabilitation hospitals (Magee Rehabilitation Hospital, Philadelphia, PA, University of Maryland Orthoapedic and Rehabilitation Institute, Baltimore, MD, Good Shepherd Rehabilitation Hospital, Allentown, PA). Matched controls (age within 5 years and gender) were recruited from the community. Sample sizes were adequate to detect an effect size (Cohen's $d$ ) of 0.7 with $80 \%$ power between groups.

Individuals were eligible for participation if $\geq 18$ years age, and included in the SCI group if determined a complete or incomplete SCI as defined by the American Spinal Injury Association impairment scale A, B, C or D [16]; were currently participating in inpatient rehabilitation following newly acquired SCI; were medically stable; and anticipated to use a MWC for at least $50 \%$ of mobility. Individuals were excluded from participating in either group if they presented with upper limb radicular symptoms, preexisting neurological conditions or health conditions that could influence upper limb function. The presence or absence of shoulder pain was not a consideration for eligibility and not included in screening criteria.

Following a review of procedures and signed informed consent, demographic data including age, gender, race, ethnicity, marital status, occupation (pre-injury for those with SCI), educational level, income level, along with injury level and mechanism of injury in those with SCI were obtained. Height and weight were extracted from the medical record for participants with SCI and measured on a calibrated scale and stadiometer in the control group. A clinical shoulder examination performed bilaterally on all participants included special tests for specific shoulder pathologies and palpation of common structures. These special tests, performed in random order, were: Neer [15] and Hawkins-Kennedy [17] impingement tests (subacromial impingement syndrome), and Speed's Test [18] (bicipital tendonitis/tendinosis). Palpation of the acromioclavicular joint, tendon insertion on the greater tuberosity, rotator cuff tendons, and biceps tendon was performed with pain report during these tests recorded as a "positive", and absence of pain a "negative" finding. Individuals with a minimum of $3 / 5$ positive clinical tests and/or tenderness to palpation were considered "positive" for shoulder impingement or tendinopathy [19]. In addition, Sulcus Sign and Load \& Shift tests assessed for shoulder joint stability were graded as "positive" or "negative" [20]. All assessments were performed with participants seated to eliminate any unnecessary transfers or repositioning [21]. Experienced physical therapists (MAF, MK) and occupational therapists 
(SKF, AS) performed all clinical measures based on a standard procedure and retraining occurred bi-annually.

\section{Study measures}

\section{Pain}

Musculoskeletal pain was assessed via the self-report Musculoskeletal Pain Survey (MPS) [13]. This survey asked participants to rate the seriousness of their pain $(0=$ not at all to $5=$ very serious $)$ and pain frequency $(0=$ never to $3=$ more than three times per week). A composite score for bilateral shoulder pain score was calculated (MPSshoulder score 0-30).

\section{Strength}

Shoulder flexion, extension, abduction, adduction, internal rotation, external rotation and horizontal adduction strength measures were acquired with standard manual muscle testing positions [22] with modification to perform all tests with participants seated. Participants with SCI stabilized with their opposite arm during testing. A handheld dynamometer measured two peak strength trials $(\mathrm{kg})$ with average used for analysis. ( $\mathrm{ICC}=0.97$ ) [23]. For normalization across participants, strength measures were divided by body weight $(\mathrm{BW})$ and expressed as a percentage $\left[\% \mathrm{BW}=\left(\right.\right.$ Force $_{\mathrm{kg}} l$ $\left.\left.\mathrm{BW}_{\mathrm{kg}}\right) \times 100\right]$.

\section{Shoulder mobility}

Two peak goniometric trials (degrees) of bilateral shoulder elevation (scapular plane), extension, external rotation $\left(90^{\circ}\right.$ shoulder abduction and $90^{\circ}$ elbow flexion), internal rotation ( $90^{\circ}$ shoulder abduction and $90^{\circ}$ elbow flexion), and horizontal adduction peak range of motion (ROM) were obtained using an established seated protocol [21, 24]. Participants with SCI stabilized with their opposite arm during testing. Shoulder goniometry reliability reported at ICC $\geq 0.94$ [25].

\section{Muscle extensibility}

Pectoralis minor muscle (PM) length, defined as the distance between the fourth rib and the coracoid process $(\mathrm{cm})$, was identified by palpation and measured with a caliper (Palpation Meter, PALM, St. Paul, MN) [26]. We obtained two trials of PM length measurement under each testing condition: resting posture and passive lengthening, with the average calculated [27]. Finley et al. [28]. established excellent psychometric properties measuring pectoralis minor muscle length under resting $(\mathrm{ICC}=0.90)$ and passive lengthening $\quad(\mathrm{ICC}=0.81) \quad$ conditions $\quad\left(\mathrm{ICC}_{2,2}=0.90\right)$.
For clinical application, extensibility is the ability of the muscle to elongate to an endpoint [29]. PM extensibility (percent change) was determined by [(passive length resting length)/resting length] $\times 100$.

\section{Tissue integrity}

A standardized series of bilateral shoulder images was obtained via musculoskeletal ultrasound (MSK-US), a multifrequency, broadband, using a 50-mm linear array transducer. MSK-US accuracy for detection of rotator cuff tears is $88.9 \%$ (95\% confidence interval $=74.1-96.21$ ) [30]. Researchers obtaining US images were trained annually by a MSK-US certified investigator (TT) and all images underwent masked quality review. The Ultrasound Shoulder Pathology Rating Scale (USPRS; maximum $=20$ per limb with 40 total) provided an impairment level score to five variables: bicipital tendinopathy, supraspinatus tendinopathy, greater tuberosity cortical surface irregularity, supraspinatus impingement and subscapularis/biceps/coracoid impingement [31]. One assessor (TT), masked to participant group and age, provided all USPRS scoring.

\section{Data analysis}

Descriptive statistics of means, standard deviations (SD) or frequencies were determined for demographics variables, pain (MPSshoulder), strength ( $\mathrm{kg}, \% \mathrm{BW}), \mathrm{ROM}, \mathrm{PM}$ extensibility and USPRS (by limb and total). Parametric assumptions (normality/skewness, homogeneity of variance) were assessed for continuous data including demographic and study measures. Based on assumptions, between group differences were analyzed with independent $t$-tests, Mann-Whitney U or Chi Square-Fishers Exact tests $(p \leq 0.05)$. As over $80 \%$ of the data met parametric assumptions, and since results from the nonparametric and parametric approaches achieved the same conclusions, results from the independent $t$-tests are reported for measures for continuous data. Pearson correlations determined by group associations of age and pain with strength $(\mathrm{kg}, \%$ BW), ROM, PM extensibility and USPRS (total). Strength of association (correlation) was weak if $r \leq 0.30$, moderate for $r=0.30-0.50$ and strong when $r>0.50$ [32]. Effect sizes were calculated for between-group measures using the following equation: Cohen $\delta=$ (mean1-mean 2$) / \mathrm{SD}_{\text {pooled }}$. An effect size of 0.2 was considered small, 0.5 was considered medium, and 0.8 was considered a large effect [32].

\section{Results}

Thirty-five volunteers (males $=32$, females $=3$ ) attending initial inpatient rehabilitation with a newly acquired SCI at 
Fig. 1 Injury characteristics of those with spinal cord injury. a Level of Injury. Yellow = Cervical; red $=\mathrm{T} 5$ above; blue $=\mathrm{T} 6$ below; green Lumbosacral. b Mechanism of injury. Yellow $=$ Fall; Red $=$ MVA; Blue $=$ GSW; Green $=$ Medical; Orange $=$ other, MVA $=$ motor vehicle accident; $\mathrm{GSW}=$ gunshot wound.

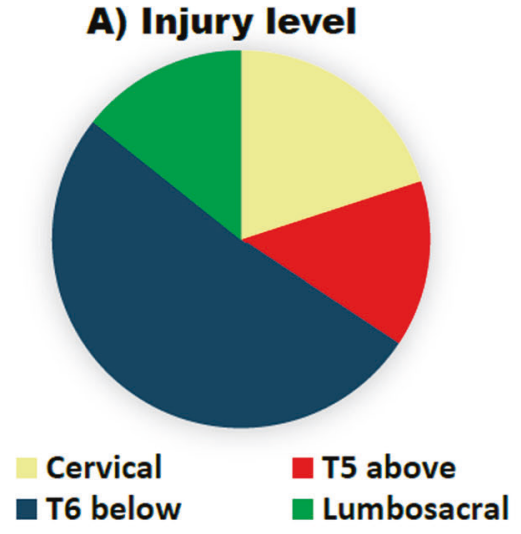

B) Mechanism of Injury

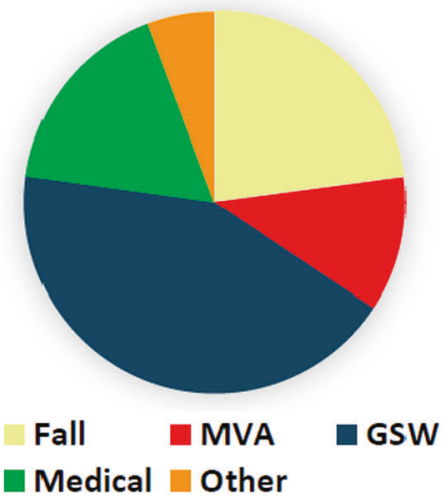

Table 1 Demographic characteristics (mean $\pm \mathrm{SD}$ ).

\begin{tabular}{lllll}
\hline & Age (years) & Height $(\mathrm{cm})$ & Weight $(\mathrm{kg})$ & Positive clinical test* \\
\hline SCI $(n=35)$ & $37.1 \pm 14.9$ & $177.3 \pm 9.1$ & $80.7 \pm 17.8$ & $31.4 \%$ \\
Control $(n=34)$ & $36.5 \pm 14.4$ & $177.6 \pm 7.4$ & $88.1 \pm 31.6$ & $11.8 \%$ \\
$p$-value & $p=0.86$ & $p=0.89$ & $p=0.23$ & $p=0.08$ \\
\hline
\end{tabular}

*Individuals with a minimum of $3 / 5$ positive clinical tests and/or tenderness to palpation were considered "positive" for shoulder impingement or tendinopathy [19] and "positive for instability with a positive sulcus sign or Load and Shift tests [20]. one of three freestanding rehabilitation hospitals and age and gender-matched uninjured peers $(n=34$, males $=1$, females $=3$ ) participated. Most participants with SCI $(>65 \%)$ demonstrated lower thoracic injury level (T6) or below with the most common mechanism of injury being gunshot wound $(43 \%)$, followed by falls (23\%, Fig. 1). No difference existed between the groups in ethnicity $(p=0.82)$, occupation $(p=0.18)$, marital status $(p=0.84)$ or annual income $(p=0.79)$. Racial differences $(p=0.03)$ were identified between the groups with more African Americans in the SCI group (SCI, $n=12$, control, $n=6$ ), while the control group included more individuals identifying as Asian (SCI, $n=0$, control, $n=6$ ). Education level differed between the groups $(p=0.18)$, the SCI group reported more individuals with high school only or less (SCI, $n=23$, control, $n=8$ ). Table 1 shows the group demographic factors. No difference existed in weight $(p=0.23)$, or height $(p=0.89)$ between the groups. In addition, no differences in clinical presentation (shoulder instability or impingement) were found $(p=0.08)$.

Shoulder pain per the MPS (non-zero score) was reported in $42.9 \%$ of those with SCI and only $17.6 \%$ in the control group $(p=0.04$, Table 2). Pain score was higher $(p=0.002)$ in SCI participants with a large between group effect size of 0.80 . The group with SCI displayed significantly lower strength ( $\mathrm{kg}$ and \% $\mathrm{BW}$ ) across all measures compared to controls, with mainly large effect sizes $(\delta=0.62-1.29)$. Shoulder mobility was reduced bilaterally in elevation, external rotation and horizontal adduction and dominant side internal rotation with moderate to large effect sizes $(\delta=0.74-1.27)$. Due to wearing a thoracolumbosacral orthosis, PM extensibility was not acquired from 4 participants with SCI. Across the remaining 31 participants with SCI, PM extensibility was reduced $(p=0.03)$ on the nondominant limb with no difference found on the dominant side $(p=0.10)$ compared to controls, however, a moderate between group effect size for each limb occurred $(\delta=0.52$ non-dominant, $\delta=0.42$ dominant). Due to adiposity and associated poor image quality, ultrasound data from two participants with SCI was not usable. No difference occurred in the USPRS score between groups $(\mathrm{SCI}=33$, control $=34)$, for dominant $(p=0.47)$, non-dominant $(p=0.19)$ or total score $(p=0.26)$.

Age and shoulder pain were not associated in the SCI group, however these two variables demonstrated a moderate, significant relationship within the control group $(r=0.36, p=0.04)$. No association with shoulder pain existed among clinical measures (strength, ROM, PM extensibility or USPRS total) in either group. Several significant $(p<0.05)$ associations with age were identified in both groups. Moderate to strong inverse correlations were found in the SCI group for all strength measures to age ( $r=-0.40$ to $-0.60, p<0.05$ ). Within the control group, relationships between strength and age were found in nondominant flexion $(r=-0.34, \quad p=0.05), \quad$ extension $(\mathrm{r}=-0.35, \quad p=0.04)$ and dominant internal rotation $(r=-0.40, p=0.02)$. For participants with SCI, ROM bilaterally in elevation, horizontal adduction and dominant side internal rotation demonstrated significant $(p<0.05)$ moderate to strong, inverse association $(r=-0.36$ to 
Table 2 Clinical Measures by group (mean $\pm \mathrm{SD})$.

\begin{tabular}{|c|c|c|c|c|}
\hline & $\mathrm{SCI}(n-35)$ & Control $(n=34)$ & $p$-value & Cohen's $\delta$ \\
\hline \multicolumn{5}{|l|}{ Musculoskeletal pain survey } \\
\hline Presence of shoulder pain (non-zero) & $42.6 \%$ & $17.7 \%$ & $p=0.02^{*}$ & - \\
\hline Shoulder pain $(\max =30)$ & $3.4 \pm 5.1$ & $0.56 \pm 1.4$ & $p=0.003^{*}$ & 0.80 \\
\hline \multicolumn{5}{|l|}{ Strength $(\% B W)$} \\
\hline Flexion_NON & $9.9 \pm 4.0$ & $14.4 \pm 4.2$ & $p<0.001 *$ & 1.09 \\
\hline Flexion_DOM & $10.1 \pm 4.3$ & $14.6 \pm 4.3$ & $p<0.001 *$ & 1.05 \\
\hline Extension_NON & $15.1 \pm 6.3$ & $22.9 \pm 6.7$ & $p<0.001 *$ & 1.20 \\
\hline Extension_DOM & $15.5 \pm 6.7$ & $22.7 \pm 5.9$ & $p<0.001 *$ & 1.14 \\
\hline Abduction_NON & $9.6 \pm 4.1$ & $13.3 \pm 3.8$ & $p<0.001 *$ & 0.94 \\
\hline Abduction_DOM & $9.7 \pm 3.9$ & $13.3 \pm 4.3$ & $p<0.001 *$ & 0.88 \\
\hline Adduction_NON & $15.4 \pm 5.6$ & $18.8 \pm 5.0$ & $p=0.01^{*}$ & 0.64 \\
\hline Adduction_DOM & $15.1 \pm 5.8$ & $18.6 \pm 5.5$ & $p=0.01^{*}$ & 0.62 \\
\hline External Rotation_NON & $11.9 \pm 3.8$ & $15.0 \pm 3.3$ & $p=0.001 *$ & 0.87 \\
\hline External Rotation_DOM & $11.4 \pm 4.0$ & $15.2 \pm 4.0$ & $p<0.001^{*}$ & 0.95 \\
\hline Internal Rotation_NON & $15.5 \pm 5.5$ & $22.7 \pm 5.7$ & $p<0.001 *$ & 1.29 \\
\hline Internal Rotation_DOM & $16.2 \pm 5.3$ & $23.2 \pm 6.2$ & $p<0.001 *$ & 1.21 \\
\hline Horizontal Adduction_NON & $11.2 \pm 3.8$ & $15.2 \pm 5.3$ & $p=0.001 *$ & 0.87 \\
\hline Horizontal Adduction_DOM & $11.4 \pm 4.6$ & $15.2 \pm 4.8$ & $p=0.001^{*}$ & 0.81 \\
\hline \multicolumn{5}{|l|}{ Range of motion (degrees) } \\
\hline Elevation_NON & $142.7 \pm 18.2$ & $159.9 \pm 8.7$ & $p<0.001^{*}$ & 1.21 \\
\hline Elevation_DOM & $142.0 \pm 15.6$ & $158.4 \pm 9.5$ & $p<0.001^{*}$ & 1.27 \\
\hline Extension_NON & $68.0 \pm 14.8$ & $68.0 \pm 10.1$ & $p=0.99$ & - \\
\hline Extension_DOM & $68.9 \pm 15.3$ & $65.8 \pm 10.5$ & $p=0.34$ & 0.23 \\
\hline External Rotation_NON & $81.7 \pm 17.9$ & $98.3 \pm 13.1$ & $p<0.001 *$ & 1.06 \\
\hline External Rotation_DOM & $84.3 \pm 18.1$ & $103.3 \pm 13.5$ & $p<0.001^{*}$ & 1.19 \\
\hline Internal Rotation_NON & $51.0 \pm 13.7$ & $56.1 \pm 14.5$ & $p=0.13$ & 0.36 \\
\hline Internal Rotation_DOM & $42.5 \pm 13.1$ & $51.8 \pm 16.7$ & $p=0.01^{*}$ & 0.62 \\
\hline Horizontal Adduction_NON & $119.9 \pm 13.4$ & $129.1 \pm 11.4$ & $p=0.004^{*}$ & 0.74 \\
\hline Horizontal Adduction_DOM & $117.2 \pm 11.2$ & $128.3 \pm 9.9$ & $p<0.001^{*}$ & 1.05 \\
\hline \multicolumn{5}{|c|}{ Pectoralis minor extensibility (\% change) } \\
\hline NON & $13.5 \pm 7.7$ & $17.3 \pm 5.5$ & $p=0.03^{*}$ & 0.57 \\
\hline DOM & $14.6 \pm 7.6$ & $17.3 \pm 4.8$ & $p=0.10$ & 0.42 \\
\hline \multicolumn{5}{|l|}{ Ultrasound Pathology Rating Score } \\
\hline USPRS_NON $(\max =20)$ & $0.33 \pm 0.65$ & $0.56 \pm 0.75$ & $\mathrm{p}=0.19$ & 0.33 \\
\hline USPRS_DOM $(\max =20)$ & $0.42 \pm 0.66$ & $0.58 \pm 1.00$ & $p=0.47$ & 0.19 \\
\hline USPRS_Total $(\max =40)$ & $0.76 \pm 1.09$ & $1.11 \pm 1.45$ & $p=0.26$ & 0.29 \\
\hline
\end{tabular}

Effect sizes Cohen's $\delta$ : small $=0.2$, medium $\delta=0.5$, large $\delta \geq 0.8$ [32].

NON non-dominant, DOM dominant.
-0.52) with age, without association shown in the control group. No correlation of PM extensibility or USPRS (by limb or total) with age existed for either group.

\section{Discussion}

This study compared musculoskeletal factors in individuals with newly acquired SCI at the rehabilitation initiation to a group of age and gender-matched uninjured individuals.
Given SCI is a sudden, unexpected event, we hypothesized no difference in musculoskeletal shoulder impairments between groups. However, our hypothesis was only partially supported. Although the shoulder tissue integrity per the USPRS was not different, those with SCI reported greater musculoskeletal shoulder pain with reduced muscle strength, shoulder mobility and muscle extensibility compared to the control participants.

Shoulder pain is a common and disabling impairment with a prevalence of $15-25 \%$ in the general population 
[33, 34]. Eriks-Hoogland et al. [12]. reported $43 \%$ of the individuals with SCI participating in inpatient rehabilitation reported shoulder pain. Similarly, $43 \%$ of our sample of people with SCI and nearly $18 \%$ of our controls reported shoulder pain. In addition to a larger percentage, the pain frequency and severity (MPS shoulder score) was higher in participants with SCI. Neither the findings of the clinical examination, nor the musculoskeletal ultrasound revealed differences between the groups, or a relationship to shoulder pain. Early onset pain following SCI was the most important predictor of pain at later time [13]. Identification of factors related to shoulder pain development and persistence is critical to determine who may develop shoulder pain and are more likely to report reduced function and independence, significant participation limitations, and decreased QoL [3, 4].

Previous evidence stated pain-free individuals with chronic SCI (mean duration of 9 years post injury) who developed shoulder pain over a three-year time period demonstrated a 10-15\% reduction in shoulder muscle strength prior to the onset of shoulder pain compared to those who remained pain-free [10]. A similar degree of gross upper limb strength deficit and associated shoulder pain development was identified by Van Drongelen et al. [13] in the early phases following SCI. In the current study, all muscle groups were significantly weaker in the group with SCI as compared to uninjured controls, with an average $13 \%$ (range 10-18\%) reduction across all muscle groups tested. However, this strength reduction was not associated with shoulder pain. In both groups strength was inversely related to age, as presented in the literature [35]. In previous studies, increasing upper limb strength by $17 \%$ predicted a $12 \%$ reduction in shoulder pain at 1 -year after discharge from inpatient rehabilitation [13]. Furthermore, increasing shoulder external rotator muscle group strength with a home exercise program exhibited the strongest mediating factor upon shoulder pain reduction for people with SCI [36]. The global shoulder strength reduction displayed by participants with newly acquired SCI provided a specific concentration focus during early rehabilitation to mitigate pain development and or progression.

Previous studies focused on passive range of motion rather than identifying potential deficits in active, voluntary motion [37]. Our study assessed active motion and compared to uninjured control participants. Individuals with newly acquired SCI displayed bilateral reduced peak shoulder elevation, external rotation and horizontal adduction with an inverse relationship to age. Although the participants with SCI were early after injury and ROM was adequate for functional tasks [38], this early motion loss was concerning as humeral elevation decreases with injury duration [37] and is a critical motion for individuals who use a wheelchair. In a typical day, wheelchair users reach overhead (above $90^{\circ}$ ) five times more often than uninjured controls [39]. A loss of humeral mobility may lead to decreased activity and community participation $[11,39]$. As in previous studies associates with shoulder pain $[3,11]$, in order to prevent further functional loss, early and active humeral mobilization in several planes should be introduced early in rehabilitation.

Individuals with chronic SCI demonstrate reduced PM extensibility compared to uninjured adults, with a strong association with duration of wheelchair use [37]. Reduced extensibility is associated with humeral elevation reduction [37]. Our group with SCI displayed reduced PM extensibility of the non-dominant side with a downward trend (moderate effect size, $\delta=0.42$ ) in the dominant limb as well. The reduced PM extensibility along with reduced active elevation found at this early phase warrants attention during rehabilitation as inflexibility of shoulder girdle soft tissues may contribute to secondary impingement for individuals with SCI [40, 41]. Periodic PM extensibility assessment with early intervention to mitigate movement impairment and shoulder pain development should also be considered.

Ultrasound examination identified supraspinatus tendinopathy and humeral cortical irregularity in $73-90 \%$ of individuals with long duration SCI [31, 42]. Brose et al. [31] reported a median USPRS score of 7 (range 2-15, nondominant) in a group of active individuals with an average SCI duration greater than 16 years, along with a significant association $(p<0.01)$ to age, weight and duration of injury. Individuals with newly acquired SCI in our study demonstrated lower mean scores with a range of only $0-4$ on the USPRS, without an association between shoulder pathology and age, or weight, in either group. Furthermore, as hypothesized, no structural shoulder difference existed between individuals with newly acquired SCI and uninjured participants indicating our sample demonstrated generally healthy shoulder structures early after injury. Previous MSK-US findings of individuals with SCI reported shoulder tissue impairments more frequently identified with commonly used special clinical tests, even $1-5$ years post injury [31, 42, 43]. As an easily administered, low cost, noninvasive method for determining shoulder impairments, MSK-US should be incorporated into routine screening following SCI for early identification and treatment of shoulder impairment.

\section{Limitations}

The study's primary limitation was the inclusion criteria individuals to possess "neurological capacity to use a manual wheelchair", which excluded individuals with higher level injuries who use power wheelchairs. Thus, we cannot generalize our results to all individuals with SCI. 
Information regarding length of stay in acute care prior to inpatient rehabilitation was not obtained and may provide insights into potential baseline differences. Though the pain quantification using the MPS shoulder has been validated in numerous studies with early SCI [11, 13], it does not provide information on task specific pain or pain interference, limiting the musculoskeletal pain description provided by our participants.

\section{Conclusions}

Our study results provided a detailed description of clinical characteristics for individuals with newly acquired SCI and the characteristic relationships with shoulder pain and age. In comparison to age and gender-matched uninjured controls, individuals with new SCI report greater shoulder pain. However, this pain did not directly associate with any clinical factors including reduced shoulder strength, range of motion, muscle extensibility, or shoulder tissue integrity. Early identified clinical impairments associate with progressive impairment and functional loss [10, 11, 37, 39] and further development and persistence of pain [13]. Early awareness and mediation of these clinical impairments may lead to improved long-term outcomes, improving the overall health and well-being of individuals with SCI.

\section{Data availability}

The authors confirm that the data supporting the findings of this study are available within the article.

Funding This work was supported by the Department of Defense, through the Spinal Cord Injury Research Program InvestigatorInitiated Research Award under Award No. W81XWH-17-1-0476.

Trial registry This trial is registered with ClinTrial.gov ID NCT03137394.

\section{Compliance with ethical standards}

Conflict of interest The authors declare that they have no conflict of interest.

Publisher's note Springer Nature remains neutral with regard to jurisdictional claims in published maps and institutional affiliations.

\section{References}

1. National spinal cord injury statistical center, facts and figures at a glance. Birmingham: University of Alabama at Birmingham; 2019.

2. Hastings J, Robins H, Griffiths Y, Hamilton C. The differences in self-esteem, function, and participation between adults with low cervical motor tetraplegia who use power or manual wheelchairs. Arch Phys Med Rehabil 2011;92:1785-8.
3. Ballinger DA, Rintala DH, Hart KA. The relation of shoulder pain and range-of-motion problems to functional limitations, disability, and perceived health of men with spinal cord injury: a multifaceted longitudinal study. Arch Phys Med Rehabil 2000;81:1575-81.

4. McCasland LD, Budiman-Mak E, Weaver FM, Adams E, Miskevics S. Shoulder pain in the traumatically injured spinal cord patient: evaluation of risk factors and function. J Clin Rheumatol 2006;12:179-86.

5. Tate DG, Kalpakjian CZ, Forchheimer MB. Quality of life issues in individuals with spinal cord injury. Arch Phys Med Rehabil 2002;83(12 Suppl 2):S18-25.

6. Veeger HE, Meershoek LS, van der Woude LH, Langenhoff JM. Wrist motion in handrim wheelchair propulsion. J Rehabil Res Dev 1998:35:305-13.

7. Subbarao JV, Klopfstein J, Turpin R. Prevalence and impact of wrist and shoulder pain in patients with spinal cord injury. J Spinal Cord Med 1995;18:9-13.

8. Ambrosio F, Boninger ML, Souza AL, Fitzgerald SG, Koontz AM, Cooper RA. Biomechanics and strength of manual wheelchair users. J Spinal Cord Med 2005;28:407-14.

9. Boninger ML, Towers JD, Cooper RA, Dicianno BE, Munin MC. Shoulder imaging abnormalities in individuals with paraplegia. $\mathrm{J}$ Rehabil Res Dev 2001;38:401-8.

10. Mulroy SJ, Hatchett P, Eberly VJ, Lighthall Haubert L, Conners S, Requejo PS. Shoulder strength and physical activity predictors of shoulder pain in people with paraplegia from spinal injury: prospective cohort study. Phys Ther 2015;95:1027-38.

11. Eriks-Hoogland I, de Groot S, Snoek G, Stucki G, Post M, van der Woude L. Association of shoulder problems in persons with spinal cord injury at discharge from inpatient rehabilitation with activities and participation 5 years later. Arch Phys Med Rehabil 2016;97:84-91.

12. Eriks-Hoogland IE, Hoekstra T, de Groot S, Stucki G, Post MW, van der Woude LH. Trajectories of musculoskeletal shoulder pain after spinal cord injury: Identification and predictors. J Spinal Cord Med 2014;37:288-98.

13. van Drongelen S, de Groot S, Veeger HE, Angenot EL, Dallmeijer AJ, Post MW, et al. Upper extremity musculoskeletal pain during and after rehabilitation in wheelchair-using persons with a spinal cord injury. Spinal Cord 2006;44:152-9.

14. Teunis T, Lubberts B, Reilly BT, Ring D. A systematic review and pooled analysis of the prevalence of rotator cuff disease with increasing age. J Shoulder Elb Surg 2014;23:1913-21.

15. Neer CS 2nd. Impingement lesions. Clin Orthop Relat Res. 1983;173:70-7.

16. Kirshblum S, Waring W 3rd. Updates for the international standards for neurological classification of spinal cord injury. Phys Med Rehabil Clin N. Am 2014;25:505-17.

17. Hawkins RJ, Kennedy JC. Impingement syndrome in athletes. Am J Sports Med 1980;8:151-8.

18. Bennett WF. Specificity of the speed's test: arthroscopic technique for evaluating the biceps tendon at the level of the bicipital groove. Arthroscopy 1998;14:789-96.

19. Hegedus EJ, Cook C, Lewis J, Wright A, Park JY. Combining orthopedic special tests to improve diagnosis of shoulder pathology. Phys Ther Sport 2015;16:87-92.

20. Clarnette RG, Miniaci A. Clinical exam of the shoulder. Med Sci Sports Exerc 1998;30:1-6.

21. Curtis KA, Roach KE, Applegate EB, Amar T, Benbow CS, Genecco TD, et al. Reliability and validity of the wheelchair user's shoulder pain index (WUSPI). Paraplegia 1995;33:595-601.

22. Kendall F, McCreary E, Provance P, Rodgers M, Romani W. Muscles: Testing and function, with posture and pain. 5th ed. Baltimore: Lippincott, Williams \& Wilkins; 2005.

23. Kolber MJ, Beekhuizen K, Cheng MS, Fiebert IM. The reliability of hand-held dynamometry in measuring isometric strength of the 
shoulder internal and external rotator musculature using a stabilization device. Physiother Theory Pract 2007;23:119-24.

24. Finley MA, Rodgers MM. Prevalence and identification of shoulder pathology in athletic and nonathletic wheelchair users with shoulder pain: a pilot study. J Rehabil Res Dev 2004;41 (3B):395-402.

25. Kolber MJ, Hanney WJ. The reliability and concurrent validity of shoulder mobility measurements using a digital inclinometer and goniometer: a technical report. Int J Sports Phys Ther 2012;7:306-13.

26. Borstad JD. Measurement of pectoralis minor muscle length: Validation and clinical application. J Orthop Sports Phys Ther 2008;38:169-74.

27. Muraki T, Aoki M, Izumi T, Fujii M, Hidaka E, Miyamoto S. Lengthening of the pectoralis minor muscle during passive shoulder motions and stretching techniques: A cadaveric biomechanical study. Phys Ther 2009;89:333-41.

28. Finley M, Goodstadt N, Soler D, Somerville K, Friedman Z, Ebaugh D. Reliability and validity of active and passive pectoralis minor muscle length measures. Braz J Phys Ther 2017;21:212-8.

29. Weppler $\mathrm{CH}$, Magnusson SP. Increasing muscle extensibility: a matter of increasing length or modifying sensation? Phys Ther 2010;90:438-49.

30. Naqvi GA, Jadaan M, Harrington P. Accuracy of ultrasonography and magnetic resonance imaging for detection of full thickness rotator cuff tears. Int J Shoulder Surg 2009;3:94-7.

31. Brose SW, Boninger ML, Fullerton B, McCann T, Collinger JL, Impink BG, et al. Shoulder ultrasound abnormalities, physical examination findings, and pain in manual wheelchair users with spinal cord injury. Arch Phys Med Rehabil 2008;89:2086-93.

32. Cohen J. Statistical power analysis for the behavioral sciences. 32nd ed. Hillsdale: Lawrence Erlbaum Associates; 1988.

33. Hasvold T, Johnsen R. Headache and neck or shoulder painfrequent and disabling complaints in the general population. Scand J Prim Health Care 1993;11:219-24.
34. Pope DP, Croft PR, Pritchard CM, Silman AJ. Prevalence of shoulder pain in the community: the influence of case definition. Ann Rheum Dis 1997;56:308-12.

35. Van Harlinger W, Blalock L, Merritt JL. Upper limb strength: study providing normative data for a clinical handheld dynamometer. PM R 2015;7:135-40.

36. Mulroy SJ, Thompson L, Kemp B, Hatchett PP, Newsam CJ, Lupold DG, et al. Strengthening and optimal movements for painful shoulders (stomps) in chronic spinal cord injury: a randomized controlled trial. Phys Ther 2011;91:305-24.

37. Finley MA, Ebaugh D. Association of pectoralis minor muscle extensibility, shoulder mobility, and duration of manual wheelchair use. Arch Phys Med Rehabil 2017;98:2028-33.

38. Namdari S, Yagnik G, Ebaugh DD, Nagda S, Ramsey ML, Williams GR Jr. et al. Defining functional shoulder range of motion for activities of daily living. J Shoulder Elb Surg 2012;21:1177-83.

39. Requejo PS, Mulroy SJ, Haubert LL, Newsam CJ, Gronley JK, Perry J. Evidence-based strategies to preserve shoulder function in manual wheelchair users with spinal cord injury. Top Spinal Cord Inj Rehabil 2008;13:86-119.

40. Burnham RS, May L, Nelson E, Steadward R, Reid DC. Shoulder pain in wheelchair athletes. The role of muscle imbalance. Am J Sports Med 1993;21:238-42.

41. Curtis KA, Tyner TM, Zachary L, Lentell G, Brink D, Didyk T, et al. Effect of a standard exercise protocol on shoulder pain in long-term wheelchair users. Spinal Cord 1999;37:421-9.

42. Finley M, Ebaugh D, Trojian T. Agreement of musculoskeletal ultrasound and clinical assessment of shoulder impairment in manual wheelchair users with various duration of spinal cord injury. Arch Phys Med Rehabil 2018;99:615-22.

43. Collinger JL, Fullerton B, Impink BG, Koontz AM, Boninger ML. Validation of greyscale-based quantitative ultrasound in manual wheelchair users: relationship to established clinical measures of shoulder pathology. Am J Phys Med Rehabil 2010;89:390-400. 